1996

\title{
Wave-Induced Chemical Chaos
}

John H. Merkin

Valery Petrov

Stephen K. Scott

Kenneth Showalter

Follow this and additional works at: https://researchrepository.wvu.edu/faculty_publications

\section{Digital Commons Citation}

Merkin, John H.; Petrov, Valery; Scott, Stephen K.; and Showalter, Kenneth, "Wave-Induced Chemical Chaos" (1996). Faculty Scholarship. 433.

https://researchrepository.wvu.edu/faculty_publications/433 


\title{
Wave-Induced Chemical Chaos
}

\author{
John H. Merkin, ${ }^{1}$ Valery Petrov, ${ }^{2}$ Stephen K. Scott, ${ }^{3}$ and Kenneth Showalter ${ }^{2}$ \\ ${ }^{1}$ Department of Applied Mathematical Studies, University of Leeds, Leeds, LS2 9JT, United Kingdom \\ ${ }^{2}$ Department of Chemistry, West Virginia University, Morgantown, West Virginia 26506-6045 \\ ${ }^{3}$ School of Chemistry, University of Leeds, Leeds, LS2 9JT, United Kingdom
}

(Received 25 September 1995)

\begin{abstract}
Spatiotemporal chaos in a two-variable, cubic autocatalator model with equal diffusivities of the species is described. The interplay between an unstable homogeneous state and propagating fronts which return the system to that state gives rise to a reinjection mechanism for chaotic behavior. Extreme sensitivity to initial conditions in both space and time and a rapid falloff of the spatial correlation function are exhibited in the chaotic regime.
\end{abstract}

PACS numbers: 82.40.Bj, 05.45.+b

Spatiotemporal chaos may arise in distributed reactiondiffusion systems by several different dynamical processes [1]. Mixed-mode chaos [2], for example, occurs when a Hopf instability of the stationary state interacts with a diffusion-induced Turing instability [3]. The stationary Turing pattern loses temporal stability when the ratio of the diffusivities for the inhibitor and the activator species becomes sufficiently small. As this ratio is further decreased, the Turing and Hopf modes increasingly mix to give rise to turbulent behavior. In this Letter, we describe spatiotemporal chaos in a reaction-diffusion system that arises from a mechanism distinctly different from this scenario or other scenarios such as phase turbulence or defect mediated turbulence $[4,5]$. We study a two-variable, cubic autocatalator model [6] in an open spatial reactorwith equal diffusion coefficients of the species. Reactiondiffusion structures emerge from an initial traveling wave, which persist indefinitely in a self-sustaining dynamical process having all the features of spatiotemporal chaos.

The governing reaction-diffusion equations for the open, unstirred autocatalator [7,8] can be written in the following dimensionless form:

$$
\begin{aligned}
& \frac{\partial \alpha}{\partial t}=\delta \frac{\partial^{2} \alpha}{\partial x^{2}}+1-\alpha-\mu \alpha \beta^{2}, \\
& \frac{\partial \beta}{\partial t}=\frac{\partial^{2} \beta}{\partial x^{2}}+\mu \alpha \beta^{2}-\phi \beta,
\end{aligned}
$$

where $\alpha$ and $\beta$ are the dimensionless concentrations of the reactant $A$ and autocatalyst $B$, respectively, and $\mu$ and $\phi$ are parameters related to the residence time and autocatalyst decay step. The parameter $\delta$ is the ratio of the diffusion coefficients $D_{A} / D_{B}$. Throughout this work we take $\delta=1$ : the equal diffusivities case.

Reaction occurs over the domain $0 \leq x \leq x_{0}$, subject to the following initial conditions:

$$
\alpha=1, \beta=\beta_{0} g(x) \text { for } t=0,
$$

where $g(x)$ is some function with compact support representing a localize input of the autocatalyst. Zero flux boundary conditions are imposed at $x=0$ and $x=x_{0}$.
The bifurcation structure of the spatially homogeneous system has the following important features (see Fig. 1). (i) There are three possible steady state solutions: the "unreacting" or extinguished state, $\left(\alpha_{s}, \beta_{s}\right)=(1,0)$, and a pair of nonzero states, $\left(\alpha_{s}^{ \pm}, \beta_{s}^{ \pm}\right)$, given by

$$
\begin{aligned}
& \alpha_{s}^{ \pm}=\frac{\mu \mp \sqrt{\mu^{2}-4 \mu \phi^{2}}}{2 \mu}, \\
& \beta_{s}^{ \pm}=\frac{\mu \pm \sqrt{\mu^{2}-4 \mu \phi^{2}}}{2 \mu \phi},
\end{aligned}
$$

which exist for $\mu \geq \mu_{\mathrm{sn}}$. The locus of saddle-node points is given by $\mu_{\mathrm{sn}}=4 \phi^{2}$. (ii) The $(1,0)$ state is a stable node for all parameter values. (iii) The $\left(\alpha_{s}^{-}, \beta_{s}^{-}\right)$ state is a saddle point. (iv) The $\left(\alpha_{s}^{+}, \beta_{s}^{+}\right)$state has a Hopf bifurcation locus emerging from a double-zero eigenvalue

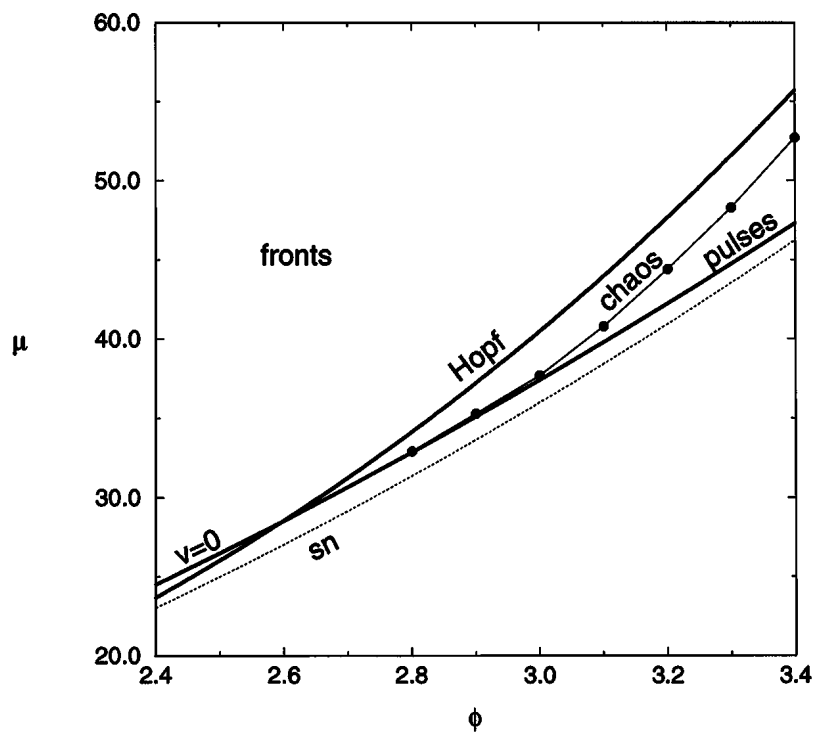

FIG. 1. Two-parameter bifurcation diagram. Simple propagating fronts are exhibited when the system is bistable, above the Hopf bifurcation line. The zero velocity line $(v=0)$ gives the lower boundary for the existence of propagating waves. The dotted line corresponds to the saddle-node bifurcation. Chaotic behavior is found in the area between the Hopf bifurcation line and the solid line with circles. 
point at $\mu=16, \phi=2$, given by

$$
\mu_{H}=\phi^{4} /(\phi-1), \quad \phi>2,
$$

with $\left(\alpha_{s}^{+}, \beta_{s}^{+}\right)$being unstable for $\mu<\mu_{H}$. The bifurcation is subcritical (unstable limit cycle emerges in $\mu>\mu_{H}$ ) over the range $2<\phi<4$, which includes the range of interest here. For $\phi>4$, the Hopf bifurcation is supercritical.

The reaction-diffusion equations, subject to the above initial conditions, allow for two different types of traveling wave solutions. First, there is a simple pulse which connects the $(1,0)$ state ahead to the same state behind the propagating wave. Such a wave will pass across the reaction domain and be extinguished at the $x_{0}$ boundary, leaving the system uniformly in the $(1,0)$ state. The second type of traveling wave connects the $(1,0)$ state ahead to the $\left(\alpha_{s}^{+}, \beta_{s}^{+}\right)$state behind, propagating with a characteristic velocity $v$. An important feature of this front structure is the damped oscillatory approach to $\left(\alpha_{s}^{+}, \beta_{s}^{+}\right)$ at the rear of the wave, which arises from the focal character of the steady state. The pulse and front solutions correspond, respectively, to homoclinic and heteroclinic connections in the $\alpha-\beta$ phase plane, and exist for particular regions of the $\mu-\phi$ parameter plane. Of particular interest in the present context is the locus in the parameter plane for which the traveling wave front solution has zero velocity, shown along with the loci for the saddle-node and Hopf bifurcations in Fig. 1.

The detailed spatiotemporal behavior of this system has been determined by direct numerical integrations of Eqs. (1a) and (1b) for various parameter values. At larger $\mu$, above the Hopf curve in Fig. 1 , the $\left(\alpha_{s}^{+}, \beta_{s}^{+}\right)$state is stable, and simple propagating fronts connecting this state and the initial $(1,0)$ state are established from suitable, nonzero inputs of autocatalyst. For parameter values above the saddle-node curve but below the zero-velocity locus, the heteroclinic front solutions have negative velocity and cannot be established from the initial input conditions studied here. Complex spatiotemporal behavior is found in the region lying between the zero velocity locus and Hopf curve. This region can be divided into two subregions, one (at lower $\mu$ ) corresponding to homoclinic pulse solutions connecting the $(1,0)$ state to itself, the other to heteroclinic front solutions connecting the $(1,0)$ and $\left(\alpha_{s}^{+}, \beta_{s}^{+}\right)$states. The focal state is a temporally unstable state of the homogeneous system, so a uniform concentration behind the front corresponding to this state cannot be established. Instead, we observe complex spatiotemporal responses in this region of parameter values, which are initiated by the first front and sustained by subsequent fronts triggered by the system itself.

An example of the spatiotemporal evolution in this region is shown in Fig. 2. In this case, an initial input of autocatalyst is supplied over a narrow region close to the origin $\left[\beta_{0} g(x)=1\right.$ for $\left.0 \leq x \leq 0.33\right]$. After a short initial transient phase, the front propagates to the right with a constant velocity ( $v \approx 0.79$ for $\mu=38$ and $\phi=3$ ) into

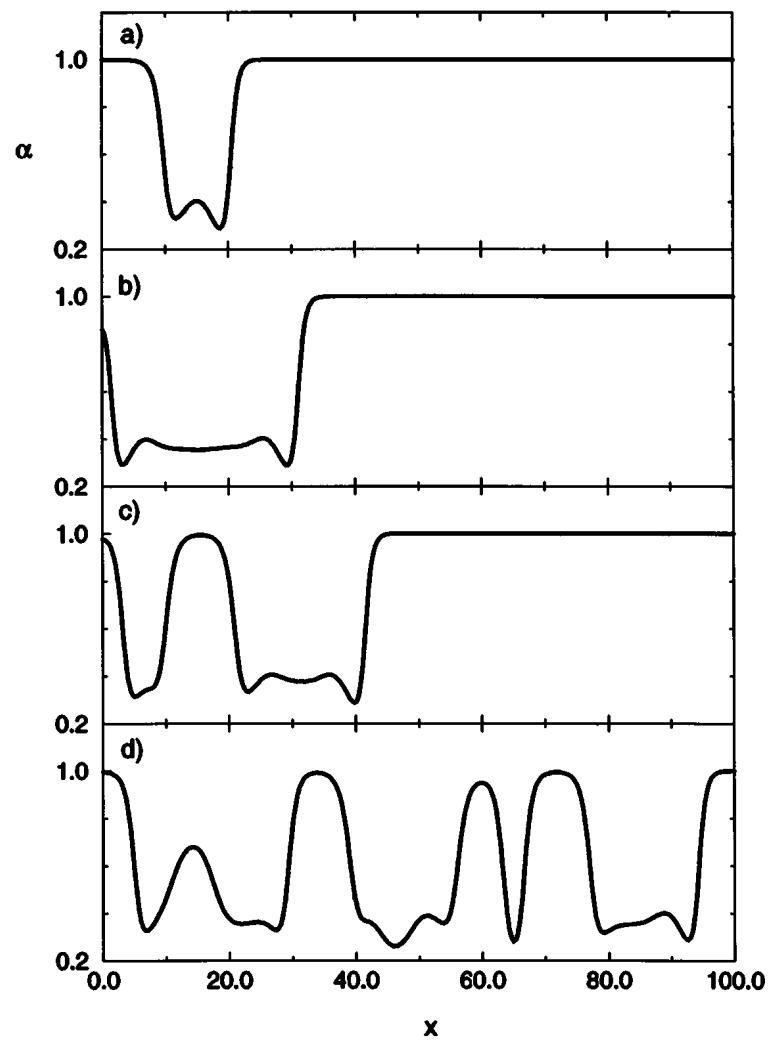

FIG. 2. Concentration profiles for $\alpha$ at different times after initiation at the left boundary: (a) $t=10$, (b) $t=24$, (c) $t=$ 38 , (d) $t=120$. The width of the reaction zone is $x_{0}=100.0$ and $\phi=3.0, \mu=38.0$. The calculation was carried out by numerical integration of the $1 \mathrm{D}$ reaction-diffusion system 1(a) and 1(b) using a finite-difference approximation with 300 grid points over the spatial domain.

the region of high $\alpha$. At the rear of the wave, there is an almost uniform region with a composition in the vicinity of the focal steady state; $\left(\alpha_{s}^{+}, \beta_{s}^{+}\right)=(0.3853,0.2049)$ for these parameter values. The system then evolves away from this state in an initially divergent oscillatory manner. The subsequent evolution cause the system to evolve toward the $(1,0)$ state, i.e., for the reaction to be extinguished locally. Figure 2(a) shows this state reestablished near the origin as the original front, now located at $x \approx 20$, propagates to the right. In the spatially uniform system, the $(1,0)$ state would represent the global attractor and would be established asymptotically. In the spatially inhomogeneous situation, however, created by the progress of the initial reaction-diffusion wave, the local region of high reactant concentration is adjacent to a region of nonzero autocatalyst concentration. This situation allows a subsequent reaction-diffusion wave to develop, propagating now to the left into the region of high $\alpha$, Fig. 2(b). Thus there is another reinjection of the system locally to the vicinity of the unstable steady state, $\left(\alpha_{s}^{+}, \beta_{s}^{+}\right)$. Also visible in Fig. 2(b) is the developing divergence from the unstable steady state over a region centered approximately on $x=15$. This gives rise to a region with $\alpha \approx 1$ and $\beta \approx 0$, shown in Fig. 2(c), into 
which a pair of fronts will subsequently propagate. Between this region and the original front, there is a further region, around $x \approx 30$, in which the system is evolving away from $\left(\alpha_{s}^{+}, \beta_{s}^{+}\right)$. The spatial asymmetry - arising as a consequence of spatially inhomogeneous initiation - is more clearly evident in this picture. Again, the system will approach the $(1,0)$ state in this region, and a pair of inwardly propagating fronts will subsequently give rise to reinjections to the vicinity of the $\left(\alpha_{s}^{+}, \beta_{s}^{+}\right)$state.

Figure 2(d) shows an example of the instantaneous $\alpha$ profile at a much later time. The original front has almost left the system on reaching $x_{0}$, but a complex and dynamic reaction-diffusion structure is sustained by repeated, spontaneous local extinction events followed by a wave-driven reinjection process.

The long-time evolution of the system is further illustrated in Fig. 3. In this space-time plot the concentration of the reactant is represented by a linear gray scale, where white corresponds to regions with $\alpha \approx 1$ and black to regions with $\alpha \approx 0$. The initial front propagates diagonally across the diagram with a constant slope characteristic of the wave velocity. The subsequent localized extinction events lead to patches near the $(1,0)$ state. These have approximately triangular shape, reflecting the constant velocity of the subsequent reaction-diffusion fronts propagating into these regions. The velocities of the subsequent fronts are similar to that of the original front.

The system does not achieve a regular spatial pattern, although some indication of a characteristic wavelength can be seen. There are phases of almost periodic evolution over localized regions, for instance, in the vicinity of the origin for $600<t<900$ and for much of the evolution at the right-hand boundary, but these are interspersed with aperiodic "bursts" of larger-scale extinction events. To test this aperiodicity for the characteristics of chaos, the single-point spatial correlation function was determined

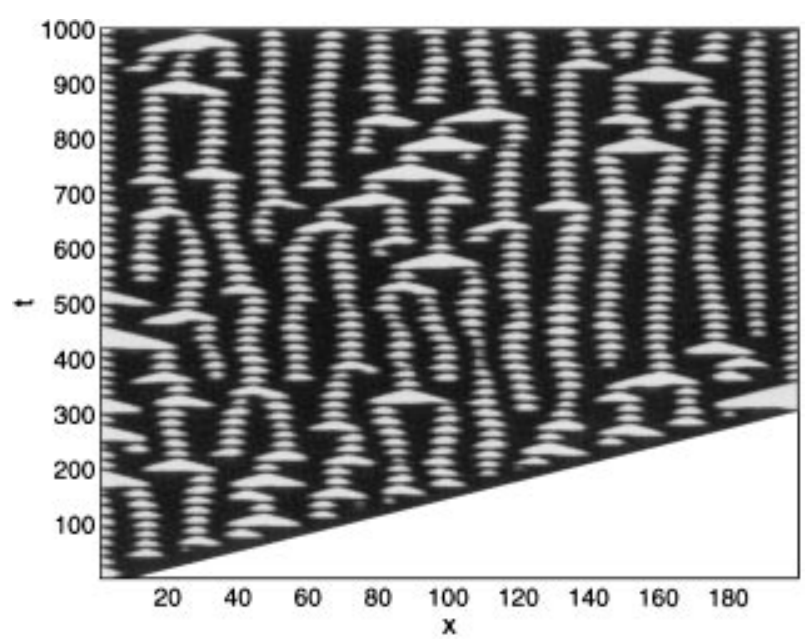

FIG. 3. Space-time plot of $\alpha$ in the chaotic regime, where white corresponds to $\alpha=1.0$ and black to $\alpha=0.0$. The front was initiated at the left boundary at $t=0$; parameter values are the same as in Fig. 2 except the width of the reaction zone is $x_{0}=200.0$. relative to the midpoint of the domain and the resulting correlation length $l_{\text {corr }}$ was evaluated. The correlation falls off rapidly, with $l_{\text {corr }}=3.2$. This value is consistent with the minimum domain length for which complex spatiotemporal responses were observed, $x_{0, \text { min }} \approx 2 l_{\text {corr }}$.

Sensitivity to initial conditions was also demonstrated. Two identical systems were computed until the posttransient behavior was established. A localized, small perturbation was then imposed on one system, and the difference in the subsequent evolution of the two systems was monitored. Not only is there a local exponential divergence of the two systems, but they also rapidly become spatially uncorrelated. These tests provide strong evidence for the existence of spatiotemporal chaos in this system for these parameter values.

The chaotic behavior is observed over a finite region in the $\mu-\phi$ parameter plane, as indicated in Fig. 1, but there are smooth qualitative changes in the character of the spatiotemporal evolution within this domain. For parameter values close to the Hopf locus, which also marks the upper boundary for the region of chaotic behavior, the system exhibits spatially extended areas in the vicinity of the $\left(\alpha_{s}^{+}, \beta_{s}^{+}\right)$state. These regions persist over long times, reflecting the near stability of this state, and are interspersed with widely spaced, localized bursts of extinction [approaching the $(1,0)$ state]. The apparent wavelength of the structures also varies across the region, reflecting the dependence of the wave front velocity and the characteristic chemical time scale for the divergence from the $\left(\alpha_{s}^{+}, \beta_{s}^{+}\right)$state on the system parameters.

A phase portrait obtained by plotting the variation of $\alpha$ with respect to $\beta$ at a single point in the domain is shown in Fig. 4. Also shown on this figure are (i) the heteroclinic connection formed by the outset of the unstable focus $\left(\alpha_{s}^{+}, \beta_{s}^{+}\right)$and the inset along the slow manifold to the stable node $(1,0)$ of the ODE system (solid, heavy curve) and (ii) the heteroclinic connection from $(1,0)$ to $\left(\alpha_{s}^{+}, \beta_{s}^{+}\right)$ formed by the constant-velocity, constant-form traveling wave front solution for this system (dashed curve). These two connections form a closed loop which plays an organizing role similar to that of homoclinic connections in the Sil'nikov mechanism for chaos $[9,10]$. The fixedpoint eigenvalues associated with the divergence from the unstable steady state $\lambda_{+}$and with the reinjection from the front connection $\lambda_{-}$have been calculated for various parameter values throughout the chaos region. Both are complex pairs in this region, with $\operatorname{Re}\left(\lambda_{+}\right)>0$ and $\operatorname{Re}\left(\lambda_{-}\right)<0$. The ratio of the magnitude of the real parts $\left|\operatorname{Re}\left(\lambda_{-}\right)\right| / \operatorname{Re}\left(\lambda_{+}\right)$decreases from infinity at the Hopf locus [where $\operatorname{Re}\left(\lambda_{+}\right)=0$ ] to a value that approaches approximately unity at the boundary between chaos and the pulse solution. Within the chaotic region of the parameter plane, therefore, the Sil'nikov criterion that the attracting manifold should have faster dynamics than the outset manifold appears to be satisfied, which is consistent with the existence of nearby chaotic orbits. This idea can be pursued further to explain, at least semiquantitatively, the 


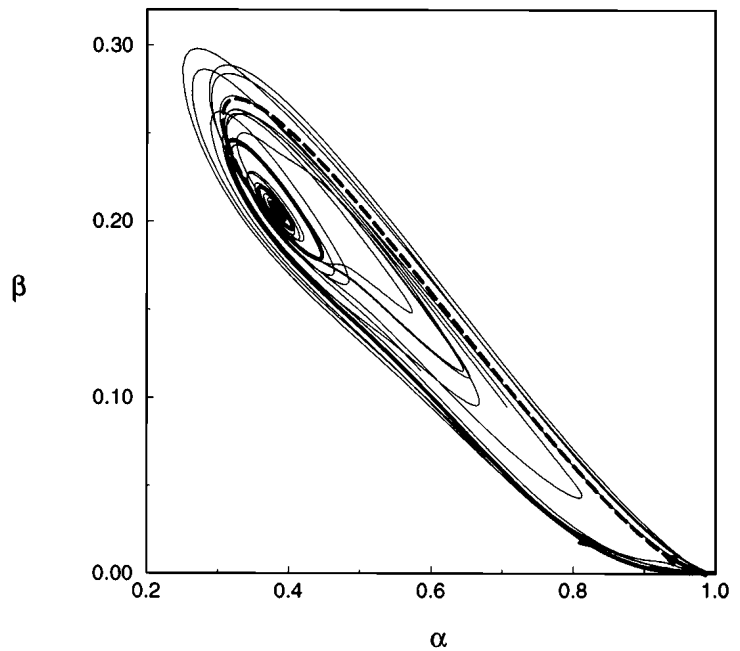

FIG. 4. The $\alpha-\beta$ phase plane for $\mu=38.0$ and $\phi=3.0$, with $x_{0}=100.0$. The thin line shows trajectories of the reaction-diffusion system taken at the middle of the reaction zone $(x=50.0)$. The heavy line represents the trajectory of the spatially homogeneous system spiraling out from the unstable focus. The heavy dashed line corresponds to the traveling wave solution of the reaction-diffusion system.

transition from chaotic behavior associated with the front reinjection mechanism to the simple, traveling pulse response. Within the region of chaos, the reinjection to the vicinity of $\left(\alpha_{s}^{+}, \beta_{s}^{+}\right)$is stronger than the outward evolution along the unstable manifold, in the sense of the relative magnitudes of the inset and outset eigenvalues. The trajectories are therefore qualitatively altered by the unstable focus with a corresponding loss of phase information. In the pulse region, however, the instability of the $\left(\alpha_{s}^{+}, \beta_{s}^{+}\right)$state is somewhat stronger and the reinjection weaker. The latter arises because the front velocity, which is involved as a multiplicative factor in the temporal eigenvalue, rapidly approaches zero as $\mu$ is decreased in the vicinity of the $v=0$ locus. Trajectories are thus more effectively repelled from the focal region and return directly to the vicinity of $(1,0)$ without experiencing the phase loss near $\left(\alpha_{s}^{+}, \beta_{s}^{+}\right)$.

The spatiotemporal chaos reported here arises from the mixing of two distinct dynamical processes. Propagating fronts convert regions near the $(1,0)$ state, where little reaction occurs, to the vicinity of the $\left(\alpha_{s}^{+}, \beta_{s}^{+}\right)$state, where both the extent of reaction and the autocatalyst concentration are significant. These regions would remain in this state were it not for the fact that it is an unstable state of the homogeneous system. Instead, the system spirals out from the unstable focal state to return to the $(1,0)$ state. The process is then repeated with fronts propagating into the new regions in the $(1,0)$ state. The behavior depends on inhomogeneous initial conditions, which in this study were in the form of an initial front. We note that the behavior is not dependent on the complete lack of reaction in the $(1,0)$ state.
Calculations were also carried out with low concentrations of autocatalyst in the input stream, and the change of the stable steady state (of the ODE system) from no reaction to slight but finite reaction had essentially no effect on the wave-induced chaotic behavior.

An essential mechanistic feature of the chaos is the wave-mediated reinjection of the system to the unstable focus, a process reminiscent of the Sil'kinov mechanism for homoclinic chaos $[9,10]$. The chaotic behavior depends on the stable manifold of the $\left(\alpha_{s}^{+}, \beta_{s}^{+}\right)$state being more strongly attracting than the unstable manifold is repelling. Phase information is lost as the system is injected into and then spirals out from the focal steady state. Chemical turbulence has also been studied by Kapral and co-workers [11,12] in the Brusselator and the complex Ginzburg-Landau equation for the case of equal diffusivities. This chaotic behavior develops in a two-dimensional medium from the interaction of vortices initiated by inhomogeneous, random initial conditions. The spatiotemporal chaos described here arises from the interplay between an unstable homogeneous state and propagating fronts which return the system to that state.

J.H.M. and S.K.S. wish to thank the Department of Chemistry, West Virginia University for their kind hospitality. K.S. is grateful to the National Science Foundation (CHE-9222616), the Office of Naval Research (N00014-95-1-0247), and the Petroleum Research Fund (29565-AC6) for supporting this research.

[1] M. C. Cross and P.C. Hohenberg, Rev. Mod. Phys. 65, 851 (1993).

[2] A. DeWit, G. Dewel, and P. Borckmans, Phys. Rev. E 48, R4191 (1993).

[3] A. Turing, Philos. Trans. R. Soc. London B 237, 37 (1952).

[4] Y. Kuramoto, Chemical Oscillations, Waves and Turbulence (Springer, Tokyo, 1984).

[5] P. Coullet, L. Gil, and J. Lega, Phys. Rev. Lett. 62, 1619 (1989).

[6] P. Gray and S. K. Scott, Chem. Engin. Sci. 39, 1087 (1984).

[7] J. E. Pearson, Science 261, 189 (1993); W. N. Reynolds, J. E. Pearson, and S. Ponce-Dawson, Phys. Rev. Lett. 72, 2797 (1994); K.-J. Lee, W. D. McCormick, J. E. Pearson, and H. L. Swinney, Nature (London) 369, 215 (1994).

[8] V. Petrov, S. K. Scott, and K. Showalter, Philos. Trans. R. Soc. London A 443, 631 (1994).

[9] L.P. Sil'nikov, Sov. Math. Dokl. 6, 163 (1965); 8, 54 (1967).

[10] P. Gaspard and G. Nicolis, J. Stat. Phys. 31, 399 (1983); P. Gaspard and X.-J. Wang, J. Stat. Phys. 48, 151 (1987).

[11] X.-G. Wu, M.-N. Chee, and R. Kapral, Chaos 1, 421 (1991).

[12] X.-G. Wu and R. Kapral, J. Chem. Phys. 94, 1411 (1991). 